\title{
Bile tolerance of Lactobacillus acidophilus LA-K as influenced by various pulsed electric field conditions
}

\author{
Olga Cueva ${ }^{1}$, Kayanush J. Aryana ${ }^{1,2^{*}}$ \\ ${ }^{1}$ School of Animal Sciences, Louisiana State University Agricultural Center, Baton Rouge, USA \\ ${ }^{2}$ Department of Food Science, Louisiana State University Agricultural Center, Baton Rouge, USA \\ Email: "karyana@agcenter.lsu.edu
}

Received 15 June 2012; revised 20 July 2012; accepted 15 August 2012

\begin{abstract}
Lactobacillus acidophilus has several health benefits and is used as an adjunct bacterium in the manufacture of cultured dairy foods. Bile tolerance is an important probiotic characteristic. Pulsed electric field (PEF) processing is non-thermal, hurdle technology which comprises of passing fluid foods between two electrodes and subjecting them to pulses of voltage for less than one second. Whether certain mild PEF conditions can enhance bile tolerance of Lactobacillus acidophilus is not known. The objective was to study the influence of certain PEF conditions on the, bile tolerance of Lactobacillus acidophilus LA-K. Lactobacillus acidophilus LA-K suspended in sterile peptone $0.1 \%$ w/v distilled water was treated with pulse widths of 3,6 and $9 \mu \mathrm{s}$, pulse periods of 10,000; 20,000 and $30,000 \mu$ and electric field strengths of 5 , 15 and $25 \mathrm{kV} / \mathrm{cm}$. The control did not receive any pulsed electric field condition. Bile tolerance was determined hourly for 16 hours. PROC GLM of the Statistical Analysis Systems (SAS) was used for data analysis. Significant differences were determined at $p$ $<0.05$. Three replications were conducted. Bipolar pulse width effect had a significant $(p<0.0001)$ influence on the bile tolerance. Bile tolerance of the control was significantly higher than the bile tolerance subjected to any of the bipolar pulse widths studied. There were no significant differences among the three different bipolar pulse widths. Pulse period had a significant $(p<0.0001)$ influence on the bile tolerance. The control and the three different pulse periods studied were significantly different from each other. The bile tolerance of the control was significantly the highest, followed by the bile tolerances subjected to $30,000 \mu$ s and $20,000 \mu$ s respectively. The bile tolerance subjected to $10,000 \mu$ s was significantly the lowest. Electric field strength had a significant ( $p$ $<0.0001)$ influence on the bile tolerance. Bile toler-
\end{abstract}

"Corresponding author. ance of the control and bile tolerance of Lactobacillus acidophilus LA-K subjected to $5 \mathrm{kV} / \mathrm{cm}$ were significantly the highest while the bile tolerance when subjected to $25 \mathrm{kV} / \mathrm{cm}$ was significantly the lowest.

Keywords: Bile Tolerance; Lactobacillus acidophilus; Pulsed Electric Field; Probiotic

\section{INTRODUCTION}

Application of high electric field strengths (typically 20 $80 \mathrm{kV} / \mathrm{cm}$ ) for short time periods (less than 1 second) to fluid foods places between two electrodes is what high intensity pulsed electric field (PEF) processing is [1]. There are several PEF factors that influence microbial inactivation, they are electric field strength, pulse wave shape, treatment time and treatment temperature.

Electric field strength, is one of the main factors influencing microbial inactivation, and is determined by the voltage $(\mathrm{kV})$ across the electrodes and the distance between the electrodes $(\mathrm{cm})$. Increasing the gap will require higher voltage to obtain the desired electric field strength [2]. The microbial inactivation increases with an increase in the electric field intensity, above the critical transmembrane potential [3]. Pore formation will occur when a certain threshold value of the transmembrane potential formed is exceeded, which was found to be in the range of $1 \mathrm{~V}$ [4]. To achieve this transmembrane potential an electric field strength above $30 \mathrm{kV} / \mathrm{cm}$ is required for most bacteria in liquid systems [5].

Pulse Waves shape can be in the form of exponential decaying pulses, square wave pulses, oscillatory pulses or bipolar pulses of electric fields. In terms of pulse shape, square wave pulses are more energy and lethally efficient than exponential decaying pulses [6]. Oscillatory pulses are the least efficient for microbial inactivation because they prevent the cell from being continuously exposed to a high intensity electric field for an extended period of time [7]. In terms of pulse polarization, bipolar pulses are more lethal than monopolar 
pulses. Bipolar pulses produce alternating changes in the movement of charged molecules, which cause a stress in the cell membrane and enhance its electric breakdown. Moreover, bipolar pulse reduces deposition of solids on the electrode surface, decreases food electrolysis, and is energy efficient [8].

Treatment time has been earlier defined as the product of the number of pulses and the pulse width [2]. The pulse width is defined as the time where the peak field is maintained for square wave pulses or the time until decay to $37 \%$ for exponential decay pulses. Pulse width influences microbial reduction by affecting the electric field intensity. Longer pulse widths decrease electric field intensity, which result in higher inactivation; however, an increase in pulse width may also result in an unwanted food temperature increase. Hülsheger et al. [9] developed a mathematical model that relates microbial survival fraction with PEF treatment time. The inactivation of microorganisms increases with an increase in treatment time [10]. The treatment time for the inactivation kinetics of tomato juice lipoxygenase by pulsed electric field was calculated with the following formula: Treatment time $=$ volume of 1 chamber $(\mathrm{mL}) /$ flow rate $(\mathrm{mL} / \mathrm{sec})^{*}$ pulse per second*number of chambers*pulse width [11].

Treatment Temperature is influenced by constant electric field strength. Constant electric field strength increases microbial inactivation as well as increases the temperature in foods. For this reason, proper cooling is necessary to maintain food temperatures far below those generated by thermal pasteurization. Vega-Mercado et al. [12] reported that $E$. coli reduction was observed to increase from 1 to $6.5 \mathrm{log}$ reduction cycles with a temperature change from $32^{\circ} \mathrm{C}$ to $55^{\circ} \mathrm{C}$.

Several health benefits of Lactobacillus acidophilus have been reported earlier [13]. Any probiotic bacterium needs to survive the bile conditions in the gastrointestinal (GI) tract before establishing in the lower GI tract to confer the health benefits upon the host. It is not known if mild PEF conditions can enhance the bile tolerance of this health beneficial bacterium. The objective was to study the influence of pulsed width, pulse period and $\mathrm{kV}$ on the bile tolerance Lactobacillus acidophilus LA-K.

\section{MATERIALS AND METHODS}

\subsection{Experimental Design}

Freshly thawed $1 \%(\mathrm{v} / \mathrm{v})$ of Lactobacillus acidophilus (F-DVS LA-K, Chr. Hansen's Laboratory, Milwaukee, WI, USA) in peptone water $(0.1 \% \mathrm{wt} / \mathrm{v})$ at room temperature $\left(21^{\circ} \mathrm{C}\right)$ was subjected to various PEF treatment conditions. The treatments were pulse widths of 3,6 , and $9 \mu \mathrm{s}$, pulse periods of $10,000,20,000$, and $30,000 \mu \mathrm{s}$, electric field strengths of 5,10 , and $15 \mathrm{kV} / \mathrm{cm}$. Control was run through the PEF equipment at $60 \mathrm{~mL} / \mathrm{min}$ without receiving any pulsed electric field treatment. Bile tolerances, were determined in the control and PEF treatment samples hourly over 16 hours of incubation. All experiments were repeated three times.

\subsection{Bile Tolerance Test}

Bile tolerance of Lactobacillus acidophilus LA-K was conducted as described earlier [14] with slight modifications. Lactobacillus acidophilus LA-K was evaluated for its ability to grow in MRS-THIO broth [MRS broth (Criterion $^{\mathrm{TM}}$, Hardy Diagnostics, Santa Maria, CA) supplemented with $0.2 \%(\mathrm{wt} / \mathrm{v})$ of sodium thioglycolate (Acros Organics, Fair Lawn, NJ)] with bile acids. Sodium thioglycolate was used in MRS broth as an oxygen scavenger to achieve microaerophilic conditions. Control and PEF treated samples were inoculated $(10 \%[\mathrm{v} / \mathrm{v}])$ into MRS-THIO broth with $0.3 \%(\mathrm{wt} / \mathrm{v})$ oxgall (bovine bile) (USBiological, Swampscott, MA) at an initial OD650 of $0.200 \pm 0.005$, and incubated under anaerobic conditions at $37^{\circ} \mathrm{C}$ for 16 hours. Absorbance in samples was measured hourly with an UV-Vis Spectrophotometer (Nicolet Evolution 100, Thermo Scientific; Madison, WI, USA) at $650 \mathrm{~nm}$. The spectrophotometer was calibrated by using MRS-THIO broth with $0.3 \%$ oxgall as blank. An average of two readings per treatment was taken, that is two cuvettes per treatment. An estimate of bacterial counts $(\mathrm{CFU} / \mathrm{mL})$ was calculated from OD650 readings using a standard curve (Figure 1).

\subsection{Statistical Analysis}

The General Linear Model (PROC GLM) of the Statistical Analysis Systems (SAS) was used to analyze the data. Differences of least square means were used to determine significant differences at $p<0.05$ for main effects and interaction effects. Significant differences were determined at $\alpha=0.05$.

\section{RESULTS AND DISCUSSION}

\subsection{Pulse Width}

The OD values at different bipolar pulse widths over the bile tolerance period of 16 hours are shown in Figure 2 . Various treatments applied are shown in Table 1. conditions are in Bipolar pulse width*hour interaction effect was significant $(p<0.0001)$ (Table 2). From hours 4 to 16 there were significant differences between the control and the three different bipolar pulse widths. Bipolar pulse width effect had a significant $(p<0.0001)$ influence on the bile tolerance (Table 2). The bile tolerance of the control was significantly higher than the bile tolerance subjected at any of the bipolar pulse widths studied. There were no significant differences among the three different bipolar pulse widths (Table 3). The bile 


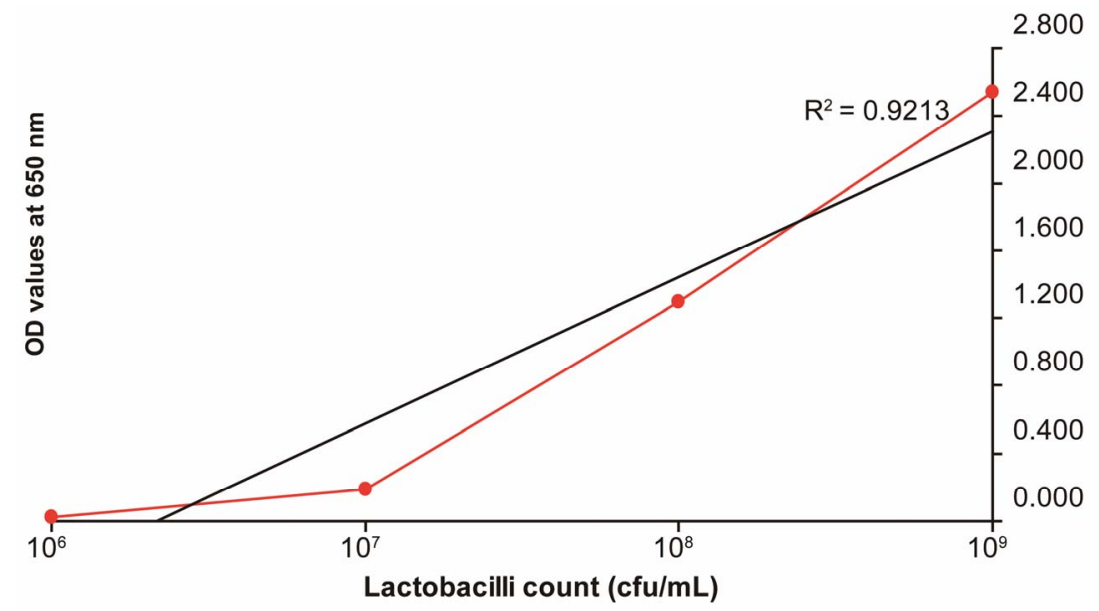

Figure 1. Standard curve for growth of LA-K in MRS-THIO broth with $0.3 \%$ oxgall.

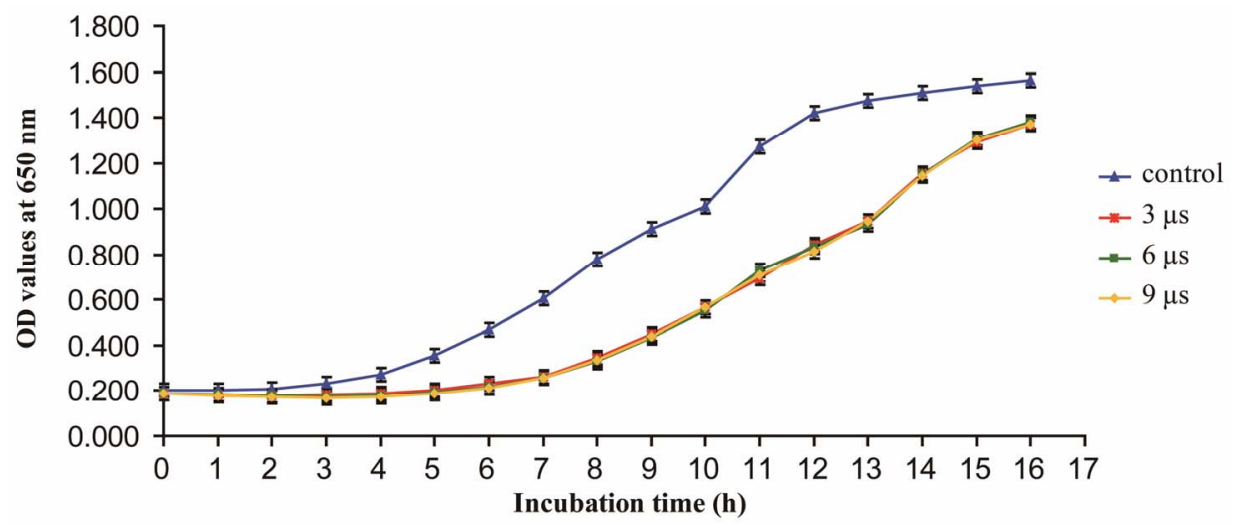

Figure 2. Pulse width effect on bile tolerance of Lactobacillus acidophilus LA-K, means \pm SE.

Table 1. PEF treatment conditions applied during the study of the influence of various pulse widths on Lactobacillus acidophilus LA-K.

\begin{tabular}{cc}
\hline Parameter & Condition \\
\hline Bipolar pulse width $(\mu \mathrm{s})$ & $3,6,9$ \\
Electric field strength $(\mathrm{kV} / \mathrm{cm})$ & 25 \\
Pulse period $(\mu \mathrm{s})$ & 10,000 \\
Delay time $(\mu \mathrm{s})$ & 20 \\
Flow rate $(\mathrm{mL} / \mathrm{min})$ & 60 \\
\hline
\end{tabular}

Table 2. Mean square (MS) and $\operatorname{Pr}>\mathrm{F}$ of pulse width, hour and their interaction for bile tolerance.

\begin{tabular}{ccc}
\hline \multirow{2}{*}{ Source } & \multicolumn{2}{c}{ Bile tolerance } \\
\cline { 2 - 3 } & MS & Pr $>$ F \\
\hline Pulse width & 1.018 & $<0.0001$ \\
Hour & 2.445 & $<0.0001$ \\
Pulse width*hour & 0.030 & $<0.0001$ \\
Error & 0.003 & \\
\hline
\end{tabular}

Table 3. Least square means for bile tolerance as influenced by pulse width.

\begin{tabular}{cc}
\hline & Bile tolerance \\
\cline { 2 - 2 } Treatment & LS Mean \\
\hline Control & $0.825^{\mathrm{A}}$ \\
$3 \mu \mathrm{s}$ & $0.546^{\mathrm{B}}$ \\
$6 \mu \mathrm{s}$ & $0.542^{\mathrm{B}}$ \\
$9 \mu \mathrm{s}$ & $0.540^{\mathrm{B}}$ \\
\hline
\end{tabular}

tolerance of different strains of Lactobacillus acidophilus isolated from human intestinal were studied by Buck and Gilliand [15]. They found that Lactobacillus acidophilus ATCC 43121 was significantly more bile tolerant than isolates $\mathrm{C} 14, \mathrm{G} 20, \mathrm{G} 5, \mathrm{H} 13, \mathrm{H} 11, \mathrm{~J} 18$ and J12. This strain required only 2 hours for the optical density to increase by 0.3 units, whereas strains $\mathrm{J} 18$ and $\mathrm{J} 12$ required 7 hours to increase.

\subsection{Pulse Period}

The OD values at different pulse periods over the bile 
tolerance period of 16 hours are shown in Figure 3. Various treatments applied are shown in Table 4. Pulse period*hour interaction effect was significant $(p<$ 0.0001) (Table 5). From hours 11 to 16 there were significant differences between the control and the three different pulse periods. However, the $10,000 \mu$ s and $20,000 \mu$ s pulse periods compared to the control, showed significant differences from hours 9 and 10 respectively. From hours 12 to 16 , among the different pulse periods, the $30,000 \mu \mathrm{s}$ pulse period was significantly higher compared to $20,000 \mu$ s which in turn was significantly higher compared to $10,000 \mu \mathrm{s}$. Bile tolerances at all different pulse periods were significantly different from each other from hours 14 to 16 . Pulse period had a significant $(p<0.0001)$ influence on the bile tolerance (Table 5). According to Table 6 the control and the three different pulse periods studied were significantly different from each other. The bile tolerance of the control was significantly the highest, followed by the bile tolerances subjected to $30,000 \mu$ s and $20,000 \mu$ s consecutively. The bile tolerance subjected to $10,000 \mu$ s was significantly the lowest. Bile resistance of Lactobacillus was arbitrarily classified into four groups according to the delay of growth [10]. These groups are: resistant strains (delay of growth $\mathrm{d} \leq 15 \mathrm{~min})$, tolerant strains $(15 \min <\mathrm{d} \leq 40$ min), weakly tolerant strains (40 $\mathrm{min}<\mathrm{d}<60 \mathrm{~min}$ ) and sensitive strains $(d \geq 60 \mathrm{~min})$ [16].

\subsection{Electric Field Strength}

The OD values at different electric field strengths over the bile tolerance period of 16 hours are shown in Figure 4. Various treatments applied are shown in Table 7. Electric field strength*hour interaction effect was significant $(p<0.0001)$ (Table 8). The bile tolerance of the control and the bile tolerance subjected to $5 \mathrm{kV} / \mathrm{cm}$ were significantly different than the bile tolerances subjected to 15 and $25 \mathrm{kV} / \mathrm{cm}$ throughout the entire incubation time period. There were no significant differences between the control and $5 \mathrm{kV} / \mathrm{cm}$ during all 16 hours period. Electric field strength had a significant $(p<0.0001)$ influence on the bile tolerance (Table 8). The bile tolerance of the control and the bile tolerance subjected to 5 $\mathrm{kV} / \mathrm{cm}$ were significantly the highest compared to 15 and $25 \mathrm{kV} / \mathrm{cm}$ (Table 9). The bile tolerance subjected to 25 $\mathrm{kV} / \mathrm{cm}$ was significantly the lowest.

Table 4. Pulsed electric field (PEF) treatment conditions applied during the study of the influence of various pulse periods on Lactobacillus acidophilus LA-K.

\begin{tabular}{cc}
\hline Parameter & Condition \\
\hline Bipolar pulse width $(\mu \mathrm{s})$ & 3 \\
Electric field strength $(\mathrm{kV} / \mathrm{cm})$ & 25 \\
Pulse period $(\mu \mathrm{s})$ & 10,$000 ; 20,000 ; 30,000$ \\
Delay time $(\mu \mathrm{s})$ & 20 \\
Flow rate $(\mathrm{mL} / \mathrm{min})$ & 60 \\
\hline
\end{tabular}

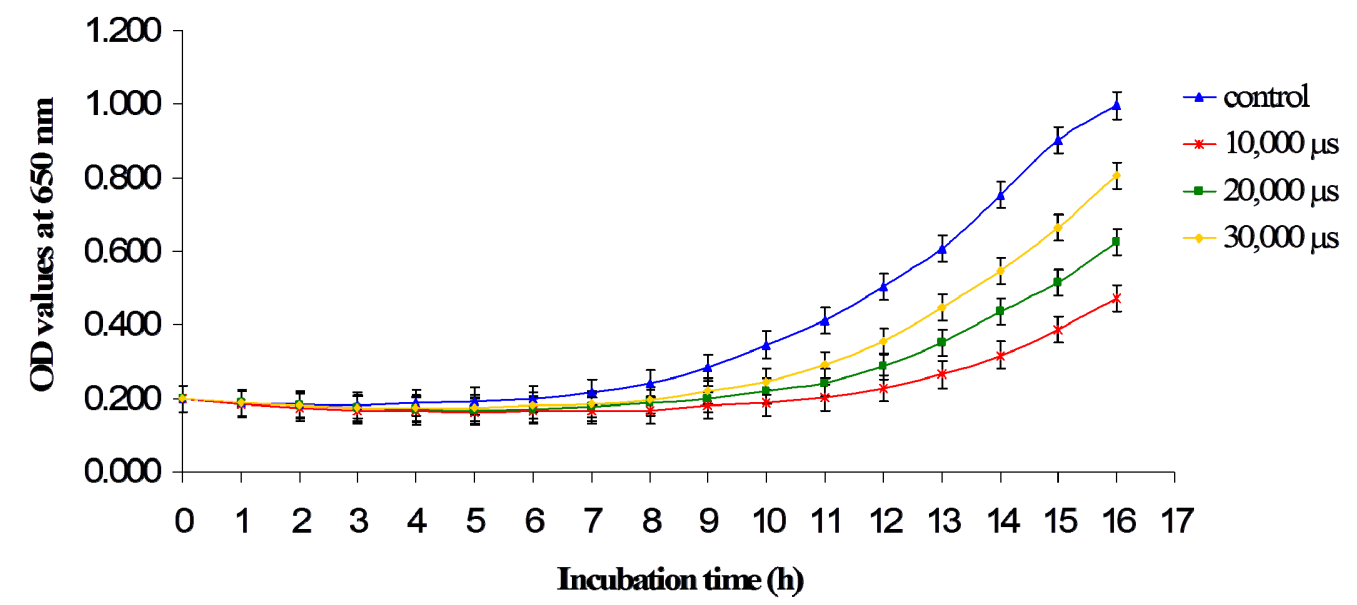

Figure 3. Pulse period effect on bile tolerance of Lactobacillus acidophilus $\mathrm{LA}-\mathrm{K}$, means $\pm \mathrm{SE}$.

Table 5. Mean square (MS) and Pr $>$ F of pulse period, hour and their interaction for bile tolerance.

\begin{tabular}{ccc}
\hline \multirow{2}{*}{ Source } & \multicolumn{2}{c}{ Bile tolerance } \\
\cline { 2 - 3 } & MS & $\operatorname{Pr}>\mathrm{F}$ \\
\hline Pulse period & 0.338 & $<0.0001$ \\
Hour & 0.473 & $<0.0001$ \\
Pulse width*hour & 0.026 & $<0.0001$ \\
Error & 0.005 & \\
\hline
\end{tabular}

Table 6. Least square means for bile tolerance as influenced by pulse period.

\begin{tabular}{cc}
\hline \multirow{2}{*}{ Treatment } & Bile tolerance \\
\cline { 2 - 2 } & LS Mean \\
\hline Control & $0.338^{\mathrm{A}}$ \\
$20,000 \mu \mathrm{s}$ & $0.223^{\mathrm{D}}$ \\
$30,000 \mu \mathrm{s}$ & $0.264^{\mathrm{C}}$ \\
\hline
\end{tabular}




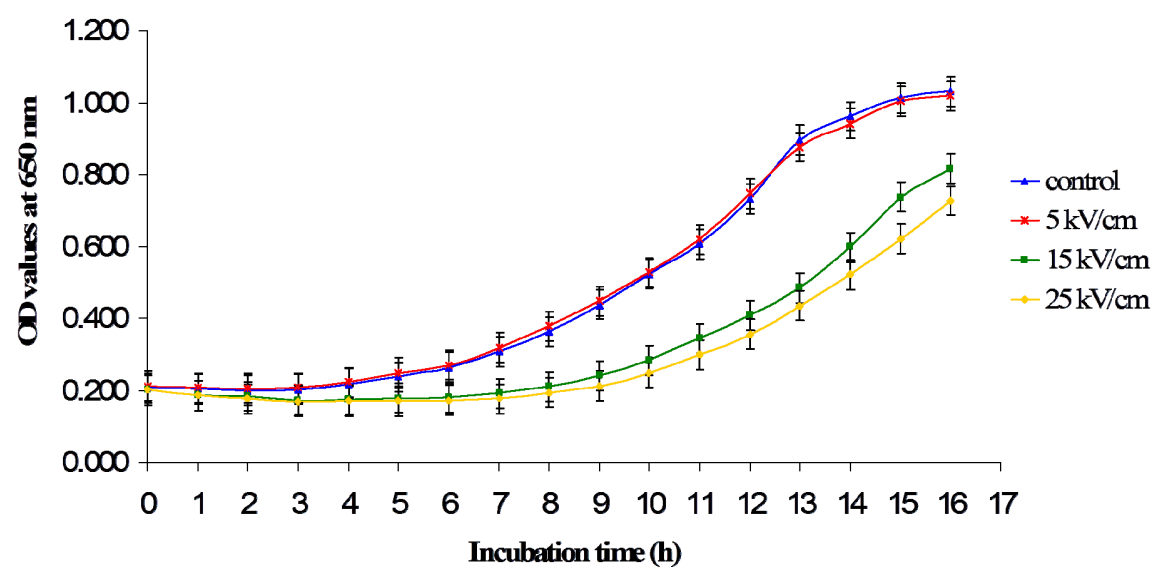

Figure 4. Electric field strength influence on bile tolerance of Lactobacillus acidophilus $\mathrm{LA}-\mathrm{K}$, means $\pm \mathrm{SE}$.

Table 7. Pulsed electric field (PEF) treatment conditions applied during the study of the influence of various electric field strengths on Lactobacillus acidophilus LA-K.

\begin{tabular}{cc}
\hline Parameter & Condition \\
\hline Bipolar pulse width $(\mu \mathrm{s})$ & 3 \\
Electric field strength $(\mathrm{kV} / \mathrm{cm})$ & $5,15,25$ \\
Pulse period $(\mu \mathrm{s})$ & 30,000 \\
Delay time $(\mu \mathrm{s})$ & 20 \\
Flow rate $(\mathrm{mL} / \mathrm{min})$ & 60 \\
\hline
\end{tabular}

Table 8. Mean square (MS) and $\operatorname{Pr}>$ F of electric field strength, hour and their interaction for bile tolerance.

\begin{tabular}{ccc}
\hline \multirow{2}{*}{ Source } & \multicolumn{2}{c}{ Bile tolerance } \\
\cline { 2 - 3 } & MS & $\operatorname{Pr}>\mathrm{F}$ \\
\hline Electric field strength & 0.582 & $<0.0001$ \\
Hour & 0.746 & $<0.0001$ \\
Electric field strength*hour & 0.021 & $<0.0001$ \\
Error & 0.005 & \\
\hline
\end{tabular}

Table 9. Least square means for bile tolerance as influenced by electric field strength.

\begin{tabular}{cc}
\hline \multirow{2}{*}{ Treatment } & Bile tolerance \\
\cline { 2 - 2 } & LS Mean \\
\hline Control & $0.494^{\mathrm{A}}$ \\
$5 \mathrm{kV} / \mathrm{cm}$ & $0.497^{\mathrm{A}}$ \\
$15 \mathrm{kV} / \mathrm{cm}$ & $0.329^{\mathrm{B}}$ \\
$25 \mathrm{kV} / \mathrm{cm}$ & $0.296^{\mathrm{C}}$ \\
\hline
\end{tabular}

\section{CONCLUSION}

Bipolar pulse width and pulse period significantly lowered bile tolerance. Electric field strength significantly influenced bile tolerance. Bile tolerance of the control LA-K and LA-K subjected to $5 \mathrm{kV} / \mathrm{cm}$ were the highest while bile tolerance LA-K subjected to $25 \mathrm{kV} / \mathrm{cm}$ was the lowest.

\section{REFERENCES}

[1] Barbosa-Cánovas, G.V., Góngora Nieto, M.M., Pothakamury, U.R. and Swanson, B.G. (1999) Preservation of foods with pulsed electric fields. Academic Press, San Diego.

[2] Zhang, Q., Barbosa-Cánovas, G.V. and Swanson, B.G. (1994) Engineering aspects of pulsed electric field pasteurization. Journal of Food Engineering, 25, 261-281. doi:10.1016/0260-8774(94)00030-D

[3] Qin, B.L., Barbosa-Cánovas, G.V., Swanson, B.G. and Pedrow, P.D. (1998) Inactivating microorganism using a pulsed electric field continuous treatment system. IEEE Transactions on Industry Applications, 34, 43-49. doi: $10.1109 / 28.658715$

[4] Zimmermann, U. and Neil, G.A. (1996) Electromanipulation of cells. CRC Press, Boca Raton.

[5] Ulmer, H.M., Heinz, V., Gänzle, M.G., Knorr, D. and Vogel, R.F. (2002) Effects of pulsed electric fields on inactivation and metabolic activity of Lactobacillus plantarum in model beer. Journal of Applied Microbiology, 93, 326-335. doi:10.1046/j.1365-2672.2002.01699.x

[6] Qin, B.L., Zhang, Q. and Barbosa-Cánovas, G.V. (1994) Inactivation of microorganisms by pulsed electric fields of different voltage waveforms. IEEE Transactions of Dielectrics and Electrical Insulation, 1, 1047-1057. doi:10.1109/94.368658

[7] Jeyamkondan, S., Jayas, D.S. and Holley, R.A. (1999) Pulsed electric field processing of foods: A review. Journal of Food Protection, 62, 1088-1096.

[8] Barbosa-Cánovas, G.V., Góngora Nieto, M.M., Pothakamury, U.R. and Swanson, B.G. (1999) Preservation of foods with pulsed electric fields. Academic Press, San Diego.

[9] Hülsheger, H., Pottel, J. and Niemann, E.G. (1981) Killing of bacteria with electric pulses of high field strength. Radiation and Environmental Biophysics, 20, 53-65. doi:10.1007/BF01323926 
[10] Hülsheger, H., Pottel, J. and Niemann, E.G. (1983) Electric field effects on bacteria and yeast cells. Radiation and Environmental Biophysics, 22, 149-162. doi:10.1007/BF01338893

[11] Min, S., Min, S.K. and Zhang, H.Q. (2003) Inactivation kinetic of tomato juice lipoxygenase by pulse electric fields. Journal of Food Science, 68, 1995-2001. doi:10.1111/j.1365-2621.2003.tb07008.x

[12] Vega-Mercado, H., Martin-Belloso, O., Chang, F.J., Barbosa-Cánovas, G.V. and Swanson, B.G. (1996) Inactivation of Escherichia coli and Bacillus subtilis suspended in pea soup using pulsed electric fields. Journal of Food Processing and Preservation, 20, 501-510. doi:10.1111/j.1745-4549.1996.tb00762.x

[13] Sanders, M.E. (2000) Considerations for use of probiotic bacteria to modulate human health. Journal of Nutrition,
130, 384S-390S.

[14] Pereira, D.I.A. and Gibson, G.R. (2002) Cholesterol assimilation by lactic acid bacteria and bifidobacteria isolated from the human gut. Applied and Environmental Microbiology, 68, 4689-4693. doi:10.1128/AEM.68.9.4689-4693.2002

[15] Buck, L.M. and Gilliland, S.E. (1994) Comparison of freshly isolated strains of Lactobacillus acidophilus of human intestinal origin for ability to assimilate cholesterol during growth. Journal of Dairy Science, 77, 29252933. doi:10.3168/jds.S0022-0302(94)77233-7

[16] Chateau, N., Deschamps, A.M. and Hadj Sassi, A. (1994) Heterogeneity of bile-salts resistance in the Lactobacillus isolates of a probiotic consortium. Letters in Applied Microbiology, 18, 42-44. doi:10.1111/j.1472-765X.1994.tb00796.x 